\title{
New Method for Previsional Design of Fabrication Process of LPG's with a Given Sensitivity
}

\author{
A. Quintela, M. A. Quintela, D. A. Gonzalez, J.M. Lázaro, P.B. García-Allende, J.M. López-Higuera \\ Photonic Engineering Group-University of Cantabria \\ Avda. Los Castios s $/ n-C . P .39005$ Santander, Spain \\ Tel: ++ 34-42-201495; Fax: ++34-42-200877_Email: quintela@ unican.es
}

\begin{abstract}
A theoretical-experimental based method able to design in advance the fabrication process for a given sensitivity (temperature, strain, surround index, ...) is proposed. Previous and proper characterization of the LPG specific fabrication technique is required.

OCIS codes:(060.2370) Fiber Optics Sensor; (050.2770) Gratings.
\end{abstract}

\section{INTRODUCTION}

The long period gratings (LPG) have been used in many photonics applications, both in optical communications and optic sensing systems. In optical communications, many devices based on LPG have been achieved and matured, even other were used for commercialization. LPG`s can be used in EDFA gain flattening and ASE equalization [1]. In the sensors field, LPG can be used for highly sensitive temperature, axial strain and refractive index sensors [2][4]. The wavelength in the resonance loss bands depend on the grating period and the effective refractive index difference between the fundamental mode and the cladding mode. External perturbations, such as strain or temperature, can modify both parameters of the LPG. In this manner, the induced variations of the resonance wavelengths can be used to implement a simple demodulation technique. Additionally, the multiple bands of the LPG can be utilized to measure simultaneously two parameters [5]. So, it possesses a highly degree of flexibility that can be used to implement various optical fiber-based sensing systems. Other advantages of the LPG used in sensor application are their easy fabrication method and that it doesn't affect the mechanical properties of the material in which they are embedded. They also present low insertion loss and low background reflections.

In the design of sensors, a custom sensitivity of the LPG to external parameters is desirable, for example to the communication applications is desirable a minimum sensitivity. So the obtaining of the sensitivity characteristics of the LPG is required to customize the design of a sensor. The dependence of the LPG characteristics on the grating period, fiber parameters and the order of the cladding mode has been theoretically studied [6] but not experimentally checked.

The objective of this paper is to present a method able to define in advance the LPG specific fa brication process for given or custom sensitivity. In order to obtain this is necessary to characterize perfectly the specific writing process of the installation. With this experimental characterization, the curves of the variation of mode resonance wavelength with the grating period are obtained for a particular fabrication conditions (UV laser power, scan speed, etc). From these results, it is possible to obtain the factor $\gamma$ for each mode and each resonant wavelength. With this factor and with the some nechanical and optical parameters of the fiber material is possible to determinate the sensitivities (temperature, strain, surround index, etc) of any resonant wavelength for a LPG written with this method. So given a sensitivity the characterization curves will give us the proper cladding mode to be used and required grating period of the LPG. The proposed method has been successfully validated using as external measured parameter the temperature. Several grating are designed fabricated and experimentally characterized showing the experimental results very good agreement with the pre-visional calculations.

\section{THEORY FOR THE SENSITIVITIES OF LPG}

The phase matching between the guided mode and the forward propagating cladding modes for a long-period grating is given by

$$
\lambda_{\text {res }}^{i}=\left(n_{\text {eff }}(\lambda)-n_{\text {cladd }}^{i}(\lambda)\right) \Lambda
$$


where $n_{\text {eff }}$ is the effective index of the fundamental core mode, $n_{\text {cladd }}^{i}$ is the effective index of the $\mathrm{i}^{\text {th }}$-order cladding mode and $\Lambda$ is the period of the core refractive index modulation or grating period.

The sensitivity of LPGs to external para meters depends on the LPG period, the order of the cladding mode to which coupling takes place and the composition of the optical fiber. The combination of the influence of these parameters permits the fabrication of LPGs with a specific sensitivity.

From(1) the analytic expression of the temperature sensitivity $d \lambda_{\text {res }} / d T$, the strain sensitivity $d \lambda_{\text {res }} / d \varepsilon$ and the surrounding refractive index $d \lambda_{\text {res }} / d n_{\text {sur }}$ are defined as [6]

$$
\begin{gathered}
d \lambda_{r e s} / d T=\lambda_{\text {res }} \cdot \gamma \cdot\left(\alpha+\Gamma_{\text {temp }}\right) \\
d \lambda_{\text {res }} / d \varepsilon=\lambda_{\text {res }} \cdot \gamma \cdot\left(1+\Gamma_{\text {strain }}\right) \\
d \lambda_{\text {res }} / d n_{\text {sur }}=\lambda_{\text {res }} \cdot \gamma \cdot \Gamma_{\text {sup }}
\end{gathered}
$$

where $\alpha$ is the thermal expansion coefficient of the fiber. In general, for silica fibers $\alpha<<\Gamma_{\text {temp. }} \gamma$ describes the waveguide dispersion and is defined by [7]

$$
\gamma=\frac{\frac{d \lambda_{\text {resn }}}{d \Lambda}}{n_{\text {eff }}-n_{\text {cladd }}^{i}}
$$

$\Gamma_{\text {temp }}, \Gamma_{\text {strain }}, \Gamma_{\text {sur }}$ describe the temperature, strain and surrounding refractive index dependences, respectively, of the waveguide dispersion and are defined by [6]

$$
\begin{aligned}
& \Gamma_{\text {temp }}=\frac{\xi_{\text {core }} n_{\text {core }}-\xi_{\text {cladd }} n_{\text {cladd }}^{i}}{n_{\text {effnicleo }}-n_{\text {effcladd }}^{(i)}} \\
& \Gamma_{\text {strain }}=\frac{\eta_{\text {core }} n_{\text {core }}-\eta_{\text {cladd }}^{i} n_{\text {cladd }}}{n_{\text {core }}-n_{\text {cladd }}^{i}} \\
& \Gamma_{\text {sur }}=\frac{u_{m}^{2} \lambda_{\text {reson }}^{3} n_{\text {sur }}}{8 \pi r_{\text {cladd }}^{3} n_{\text {cladd }}\left(n_{\text {core }}-n_{\text {cladd }}^{i}\right)\left(n_{\text {cladd }}^{2}-n_{\text {sur }}^{2}\right)^{3 / 2}}
\end{aligned}
$$

where $\xi_{\text {sore }}, \xi_{\text {cladd }} \mathrm{y} \eta_{\text {core }}, \eta_{\text {cladd }}$ are the thermooptic and elastooptic coefficients of the core and cladding materials, respectively. $u_{m}$ is the $\mathrm{m}^{\text {th }}$ root of the zeroth-order Bessel function of the first kind. $r_{\text {cladd }}$ and $n_{\text {cladd }}$ are the radius and refractive index of the fiber cladding, respectively.

From (2) and (4), it can be deducted that the sensitivity of LPGs to temperature and surrounding refractive index is determined predominantly by the product $\gamma \cdot \Gamma_{i}$ where $i$ is the specific external parameter. And from (3), the strain sensitivity of LPGs depends on the product $\gamma \cdot\left(1+\Gamma_{\text {strain }}\right)$. The term $\Gamma_{i}$ is the material contributions and it is related with the core and cladding refractive index. This contribution depends specially on the composition of the fiber and the order of the cladding mode. The term $\gamma$ is the waveguide contribution. This factor has determinant influence on the LPG sensitivity because it is directly co-related with $d \lambda_{r e s} / d \Lambda$.

\section{METHOD EXPLANATION}

The first step in order to obtain the curves that permit know the different sensitivities of the LPG is writing some LPGs . With that the real dependence of $\lambda_{\text {res }}^{i}$ with $\Lambda$ will be obtained for the LPGs written with the installation. So it is necessary to write LPGs with different periods but with the same conditions of fabrication (laser power, number of periods, duty cycle, scan speed, etc). The number of LPG written was 32, with periods between $140 \mu \mathrm{m}$ and 450 $\mu \mathrm{m}$ All LPG were writing in hydrogenated standard telecommunications fiber using a continuous wavelength argon 


\section{TuE15.pdf}

ion laser at $244 \mathrm{~nm}$. The fabrication technique used was point-to point with a continuous scan speed and a synchronize shutter. A microscope objective was using in order to make the UV spot as small as possible. As the numbers of periods was the same in each LPG, the length of each LPG was different. In Fig. 1 it is shown the results obtained from the measures and a simulation that approach the experimental measures. The simulation have been done with the software Ifograting, using the parameters of the used fiber, in order to obtain the effectives index of the $\mathrm{i}^{\text {th }}$-orders cladding mode. A very good agreement between the calculated (continuous lines) and the experimentally measures (discrete points) on Fig. 1 can be deducted.

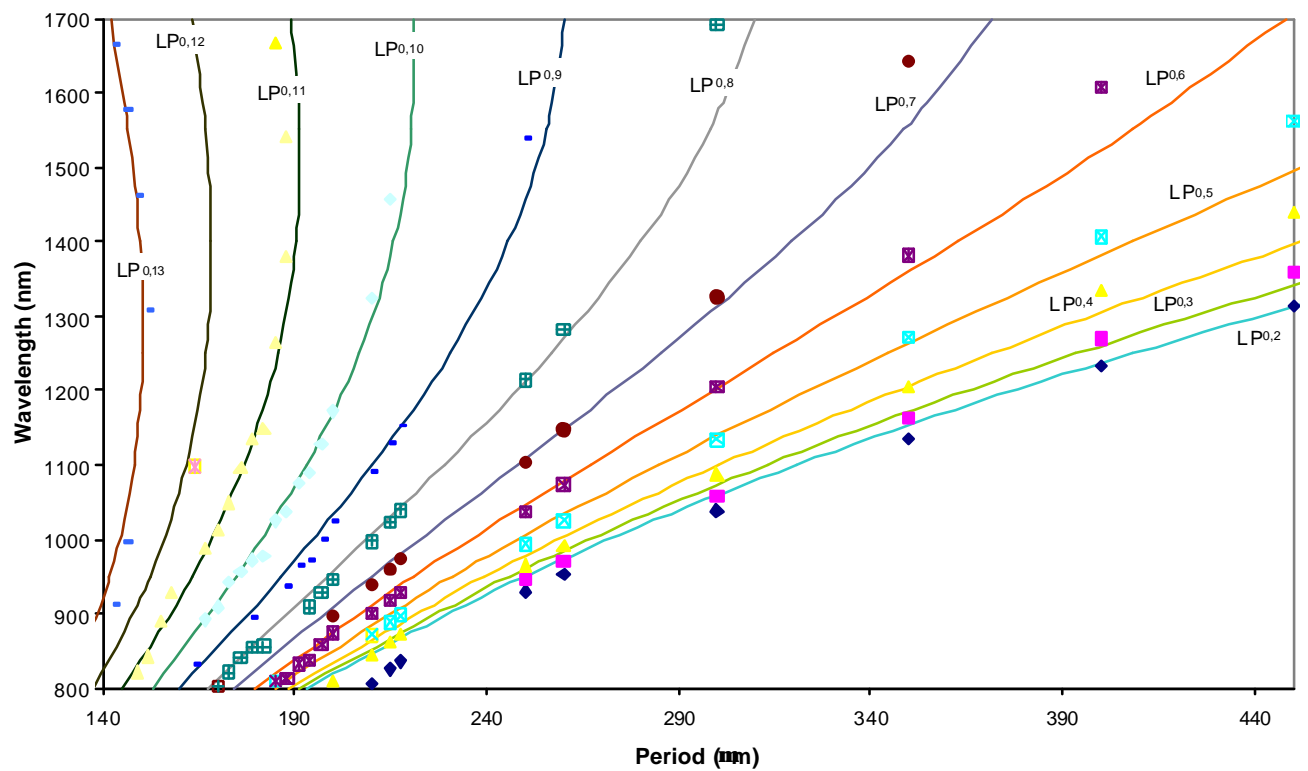

Fig. 1.Experimental(continuous line) and simulated (discrete points) of several cladding mode res onance wavelengths with the LPG period

Now, using the results included in the Fig. 1, the parameter ?, of the equation (5), for each cladding mode in function of the wavelength is calculated and their results summarized in the Fig. 2. These results are valid for any LPG write using the characterized fabrication process. With its curves and the equations (2),(3),(4) the different sensitivities for each coupled cladding mode can be obtained.

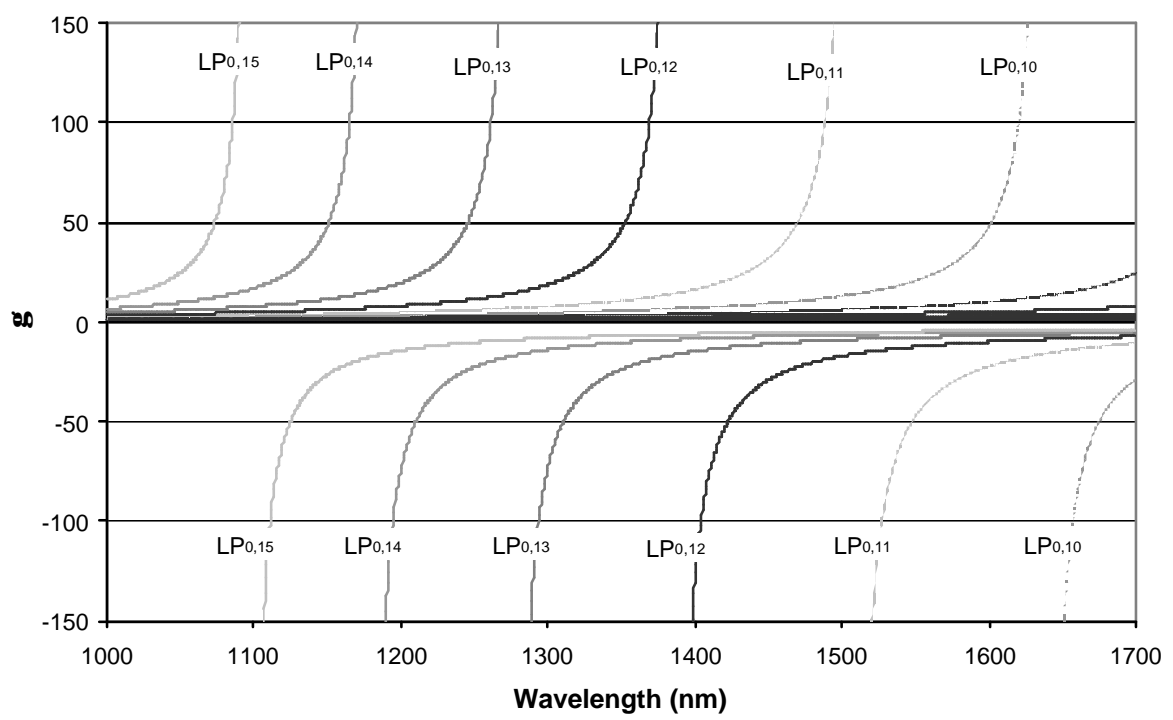

Fig. 2. Value of " $\gamma$ " obtained from the characterization of $L P G$ witting process 
So in the moment that is desired to write a LPG with a required sensitivity in a spectral region the procedure to do is the follow. First, from the curves of sensitivity versus wavelength the appropriate cladding mode that offers the desired sensitivity at the wanted spectral region is determined. Knowing the cladding mode at the resonant wavelength and using the curves of Fig. 1 it is obtained the period of the LPG wanted.

\section{METHOD CHEKING}

In order to validate the proposed method some LPG were designed, fabricated and heated between 0 an $70^{\circ} \mathrm{C}$ in $10^{\circ} \mathrm{C}$ incremental in a climatic chamber in our laboratory. In the Fig. 3 are summarized the experimental results of the relative wavelength shift of some loss peak and the curve o sensitivities predicted using the method explained above. As can be deducted the agreement between the experimental results and the predicted sensitivities are very good and the de method was successfully validated.

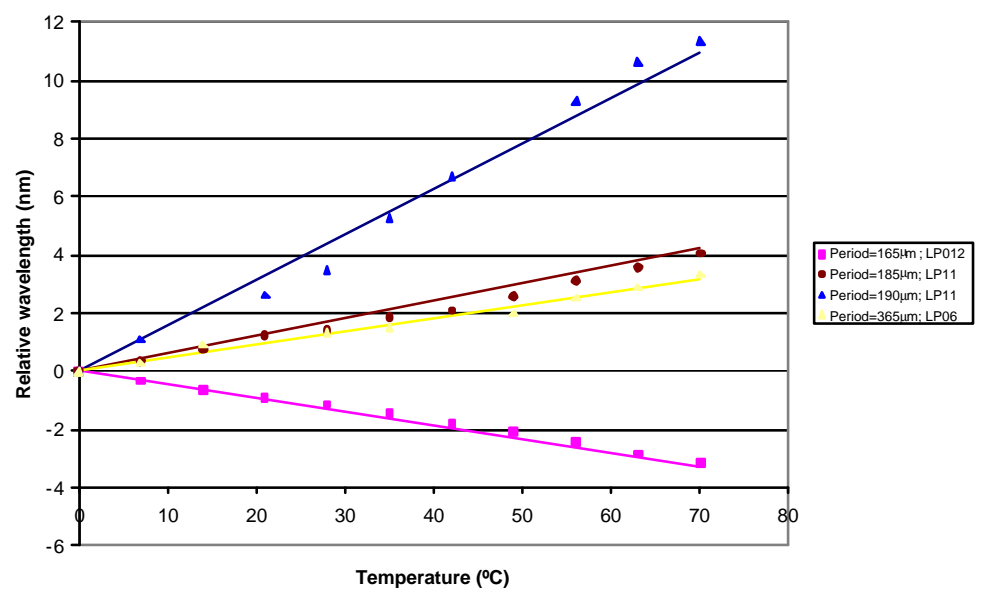

Fig. 3.Predicted an real temperature sensitivities of several LPG

\section{CONCLUSIONS}

A method able to determine in advance the proper fabrication process for a given sensitivity has been proposed. With a previous characterization of the LPG writing process is possible to know a priori the value of the different sensitivities (temperature, strain, surrounding index, etc) that will have the LPG. The parameter ?, that plays a key role in the different sensitivities, is predicted from the experimental data obtained in the characterization of the LPG writing for each kind of fiber. This parameter was experimental obtained for any cladding mode and any wavelength. Using this curve the definition of the period of the grating and of the fabrications parameters for the required sensitivity are obtained. This fabrication method has been validated fabricating several gratings and doing experimental measurement of their temperature sensitivity.

\section{ACKNOWLEDGEMENTS}

This work has been co-supported by the Spanish TEC2004-05936-C02 and TEC2005-08218-C02-02 projects.

\section{REFERENCES}

[1] R. Kashyap, Fiber Bragg Gratings Harcourt Brace \& Company, New York, 1999.

[2] W. James and R. P. Tatam, "Optical fibre long-period grating sensors: characteristics and application", Measurement Science and Technology, Vol. 14, R49-R59, 2003.

[3] J. M. López-Higuera, Editor. Handbook of OpticalFiber sensing Technology, Ed. Wiley \& Sons, 2002.

[4] X. Shu, T. Allsop, B. Gwandu, L. Zhang and I. Bennion, "High -temperature sensitivity of long-period gratings in B-Ge codoped fiber", IEEE Photonics Technology Letters, Vol. 13, No. 8, 818820, 2001.

[5] V. Bhatia, "Applications of long-period gratings to single and multi-parameter sensing", Optics Express, Vol. 4, No. 11, 457 466, 1999.

[6] X. Shu, L. Zhang and I. Benn, "Sensitivity characteristics of long-Period fiber grating", Journal of Lightwave Technology, Vol. 20, No. 2, $255-266,2002$.

[7] T. W. Macdougall, S. Pilevar, C. W. Haggans and M. A. Jackson, "Generalized expression for the growth of long period gratings", IEEE Photonics Technology Letters, Vol. 10, No. 10, 1449-1451, 1998. 